\title{
Intellectual ability and cortical development in children and adolescents
}

\author{
P. Shaw ${ }^{1}$, D. Greenstein ${ }^{1}$, J. Lerch ${ }^{2}$, L. Clasen $^{1}$, R. Lenroot ${ }^{1}$, N. Gogtay ${ }^{1}$, A. Evans ${ }^{2}$, J. Rapoport ${ }^{1} \&$ J. Giedd ${ }^{1}$
}

Children who are adept at any one of the three academic ' $R$ 's (reading, writing and arithmetic) tend to be good at the others, and grow into adults who are similarly skilled at diverse intellectually demanding activities ${ }^{1-3}$. Determining the neuroanatomical correlates of this relatively stable individual trait of general intelligence has proved difficult, particularly in the rapidly developing brains of children and adolescents. Here we demonstrate that the trajectory of change in the thickness of the cerebral cortex, rather than cortical thickness itself, is most closely related to level of intelligence. Using a longitudinal design, we find a marked developmental shift from a predominantly negative correlation between intelligence and cortical thickness in early childhood to a positive correlation in late childhood and beyond. Additionally, level of intelligence is associated with the trajectory of cortical development, primarily in frontal regions implicated in the maturation of intelligent activity ${ }^{4,5}$. More intelligent children demonstrate a particularly plastic cortex, with an initial accelerated and prolonged phase of cortical increase, which yields to equally vigorous cortical thinning by early adolescence. This study indicates that the neuroanatomical expression of intelligence in children is dynamic.

Structural neuroimaging studies generally report a modest correlation $(r=0.3)$ between psychometric measures of intelligence and total brain volume ${ }^{6}$. Links between intelligence and specific regions of the brain may vary according to developmental stage: the anterior cingulate in children ${ }^{7}$, the orbitofrontal and medial prefrontal cortex in adolescents ${ }^{8}$, and the lateral prefrontal cortex in older adults ${ }^{9}$. Most previous studies infer developmental processes from purely cross-sectional data, an endeavour fraught with methodological complications ${ }^{10}$. Only one longitudinal study has linked cortical development with cognitive variation, demonstrating greater cortical thinning in the left dorsal frontal and parietal regions among children who gained more in a measure of verbal intelligence ${ }^{5}$. However, this study was limited by its small sample size $(n=45)$, narrow age range (5-11 yr), and consideration of only linear cortical change, whereas brain development generally follows more complex growth patterns ${ }^{7,11}$.

We characterized brain development from childhood to adulthood in a large group of typically developing subjects $(n=307)$, the majority of who had prospectively acquired repeated neuroanatomic scans (see the Methods). Subjects were stratified on the basis of Wechsler intelligence scales, which give a standardized 'intelligence quotient' (IQ) based on subtests assessing verbal and non-verbal knowledge and reasoning ${ }^{12}$. We examined the thickness of the cortex throughout the entire cerebrum, as it is a sensitive index of normal brain development ${ }^{5,13}$, using a fully automated technique, and have validated these measurements by expert manual determination of cortical thickness and population simulations ${ }^{14,15}$. We reasoned that the trajectory of cortical development in children stratified on the basis of IQ would differ primarily in the prefrontal cortex, which has both structural and functional correlations with intelligence. The institutional review board of the National Institutes of Mental Health approved the research protocol, and written informed consent and assent were obtained from parents and children, respectively.

We estimated Pearson's correlations between IQ and cortical thickness for all subjects (each subject contributing one scan), and found modest positive correlations throughout most of the frontal, parietal and occipital cortex, and similarly modest negative correlations in the anterior temporal cortex (Fig. 1 and Supplementary Table 1). Throughout most of the cerebral cortex, the correlations were not significant at an unadjusted $P<0.05$.

Dividing the sample into different age groups, however, revealed notable age-related changes. A predominantly negative correlation between IQ and cortical thickness in the early childhood group contrasted with later positive correlations, which peaked in late childhood, but were present in an attenuated form in the adolescent and early adult groups. The change in the valence of the correlation between IQ and cortical thickness was significant between the young and late childhood groups throughout the prefrontal cortex, and the

a

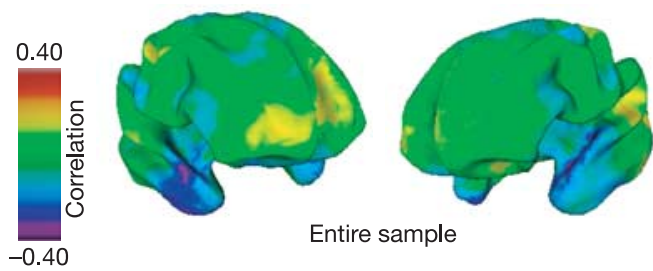

b
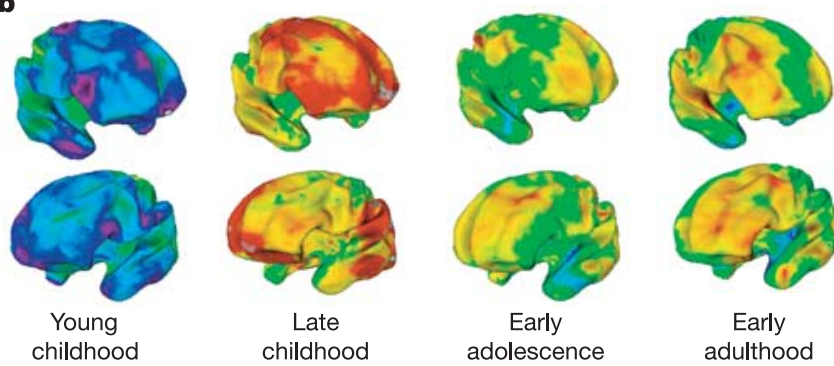

Figure 1 | Correlations between IQ and cortical thickness. a, Pearson's correlations for all 307 subjects were generally positive and modest $(P>0.05)$, with $r$ between 0 and 0.10 (green/yellow), except in the anterior temporal cortex (which showed a negative correlation, with $r$ between 0 and -0.1 ; blue/purple). $\mathbf{b}$, Correlations in different age groups showed that negative correlations were present in the youngest group, indicating that higher IQ was associated with a thinner cortex particularly in frontal and temporal regions. The relationship reverses in late childhood, with most of the cerebral cortex correlating positively with IQ. 

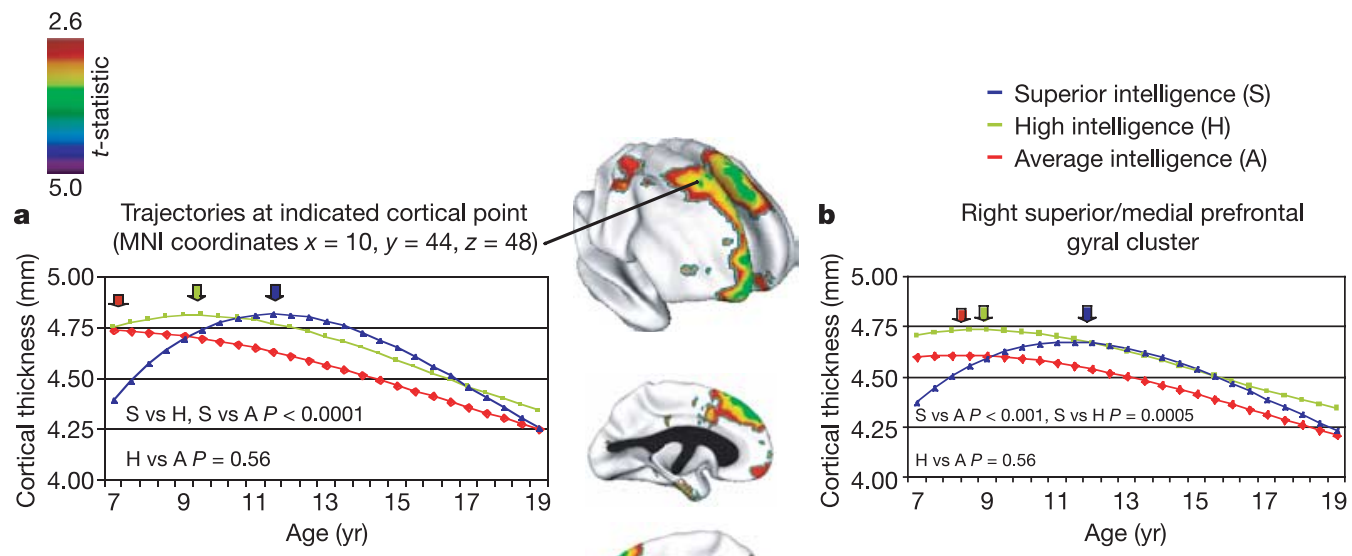

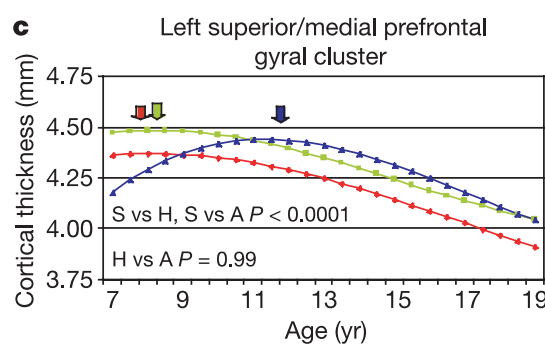

Figure 2 | Trajectories of cortical change. The brain maps (centre panel) show prominent clusters where the superior and average intelligence groups differ significantly in the trajectories of cortical development ( $t$-statistic maps show areas of significant interaction between these IQ groups and the cubic age term). a, Graph showing the trajectories at the cortical point of maximum trajectory difference in the right superior frontal gyrus (point

left superior/middle temporal gyri. These age groups did not differ in gender composition $\left(\chi^{2}=2.76 ; P=0.62\right)$ or mean IQ $\left(F_{3,303}=1.58 ; P=0.19\right)$, and there was no significant gender difference in the correlation between cortical thickness and IQ.

We further characterized the development of the relationship between intelligence and cortical morphology using linear mixedmodels, which allowed inclusion of all 629 scans. In the determination of cortical thickness, there was a significant interaction between IQ and age terms in the prefrontal cortex, suggesting that the relationship between cortical thickness and IQ varies with age (specifically cubic and quadratic age terms; see the Supplementary Figure).

To explore this interaction, the sample was split into three IQ groups: superior, high and average intelligence. Prominent clusters of cortical points showing differences in cortical development between the intelligence groups lay bilaterally within the superior frontal gyri extending into the medial prefrontal cortex, and to a lesser extent in the middle and orbitofrontal cortices (Fig. 2). In each of these clusters, the trajectories for the local point of maximum trajectory difference and for the entire cluster were similar: the superior intelligence group started from a relatively thinner cortex, but then showed a marked increase in cortical thickness peaking at $\sim 11 \mathrm{yr}$. In contrast, the average intelligence group showed either a steady decline in cortical thickness throughout the age period covered (in orbitofrontal areas), or a short initial increase in cortical thickness which peaked at $\sim 7-8 \mathrm{yr}$ (in superior frontal gyri). The trajectories of the high intelligence group followed an intermediate pattern, more strongly resembling the pattern of the average intelligence group, with no significant differences between these two groups in the clusters shown in Fig. 2 (all $P>0.10$ ).

Different developmental trajectories were also prominent in the posterior left hemisphere between the superior and average intelligence groups, specifically within the left middle prefrontal and inferior temporal gyri, and to a lesser extent the angular gyrus. The right hemisphere outside the frontal lobes showed trajectories of cortical development that did not differ significantly between groups.

An overall decline in cortical thickness was noted in all groups, present either throughout the age period covered (average intelligence group) or starting by late childhood (high intelligence) or early adolescence (superior intelligence). Velocity curves derived using a first-order differential of the fitted cubic growth curves illustrate that the superior intelligence group had the most rapid rate of cortical thinning, whereas the high and average intelligence groups had similar, but slower, rates (Fig. 3). Thus, the relatively rapid increase in cortical thickness in the superior intelligence group was followed by a more rapid thinning.

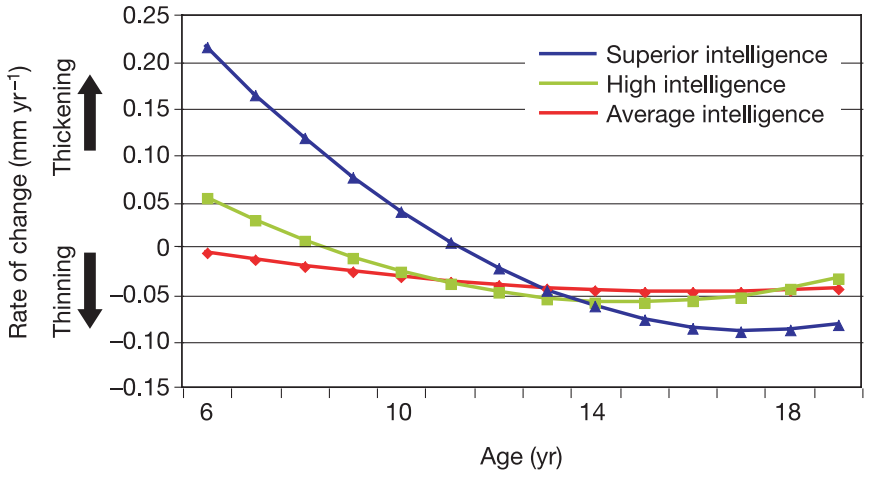

Figure 3 Rate of change in cortical thickness. The rate of change for the cluster of cortical points in the right superior and medial frontal gyrus, which showed a significant trajectory difference. Positive values indicate increasing cortical thickness, negative values indicate cortical thinning. The point of intersection on the $x$ axis represents the age of maximum cortical thickness (5.6 $\mathrm{yr}$ for average, $8.5 \mathrm{yr}$ for high, and $11.2 \mathrm{yr}$ for the superior intelligence group). 


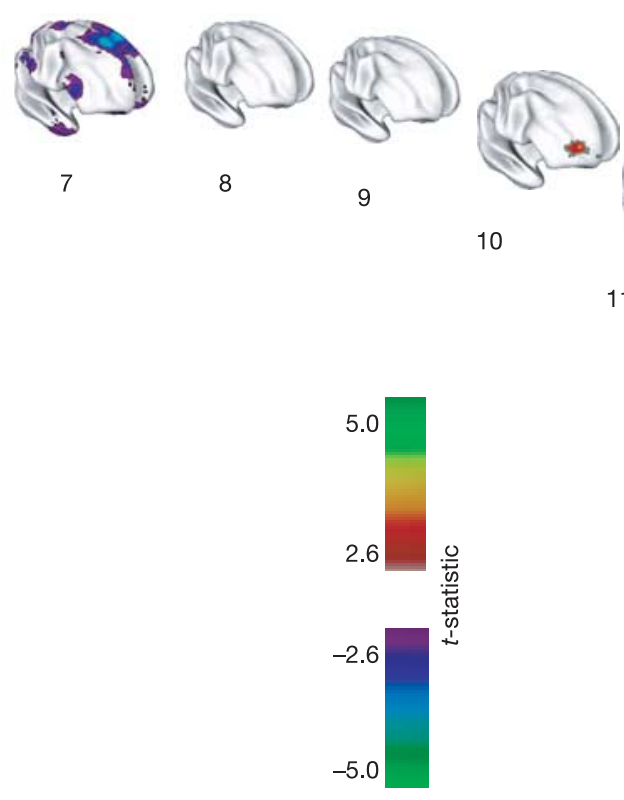

Figure 4 Developing differences in cortical thickness between the superior and average intelligence groups. Group differences are represented by $t$-statistics $(t>2.6)$, and show that the superior intelligence group has a thinner superior prefrontal cortex at the earliest age (purple
To illustrate the development of differences in cortical thickness between the superior and average intelligence groups, statistical maps representing group differences in the height of the developmental curves at each age were estimated from 7-16 yr (Fig. 4). Initially, the superior intelligence group had a relatively thinner cortex in superior prefrontal gyri, but then showed a rapid increase in cortical thickness. By $11 \mathrm{yr}$, regions of thicker cortex became apparent in the superior intelligence group-initially in anterior portions of the right superior and middle frontal gyri, spreading to involve more posterior regions of the right prefrontal cortex and the left superior and middle frontal gyri. By late adolescence, the accelerated rate of cortical loss in the most intelligent group leads to decreased regional differences.

The intelligence groups did not differ significantly in handedness or gender composition, but did in socio-economic status $\left(F_{2,291}=14.1 ; \quad P<0.001\right)$, which was correlated with IQ $(r=-0.35 ; P<0.01)$. In the frontal clusters, where trajectories were most closely tied to intelligence, none of these variables contributed significantly to the final polynomial regression model (all $P$ values $>0.1$ ).

Thus, we have demonstrated that level of intelligence is related to the pattern of cortical growth during childhood and adolescence. The differing trajectories of cortical change are most prominent in the prefrontal cortex, congruent with functional magnetic resonance imaging ( $\mathrm{fMRI}$ ) studies showing that activation of the lateral prefrontal cortex is common to a range of intelligence tests, and that the magnitude of frontal cortical activation correlates highly with intelligence ${ }^{16,17}$.

Our longitudinal structural MRI images provide adequate resolution to describe an in vivo change in cortical thickness, but the nature of the underlying cellular events is largely unknown. A determinant of cerebral lamination in utero and perinatally is the emergence and resolution of the subplate, which contains neurons, developing cortical afferents and their synapses ${ }^{18,19}$. Proliferation of myelin into the peripheral cortical neuropil in childhood and adolescence is another possible mechanism influencing cortical thickness ${ }^{5,20}$. Additionally, the formation and usage-dependent selective elimination of synapses ${ }^{21}$,

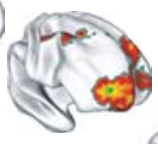

11

12

13

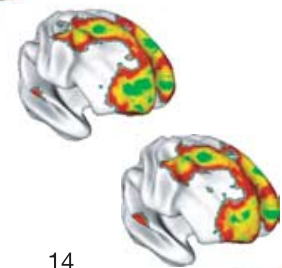

15

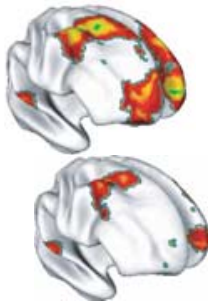

regions). There is then a rapid increase in cortical thickness (red, green and yellow regions) in the superior intelligence group, peaking at age 13 and waning in late adolescence.

which help to create and sculpt neural circuitry including those supporting cognitive abilities ${ }^{22}$, may contribute to changing cortical dimensions. The prefrontal cortex shows relatively late structural ${ }^{11}$ and metabolic ${ }^{23}$ maturation, and the prolonged phase of prefrontal cortical gain in the most intelligent might afford an even more extended 'critical' period for the development of high-level cognitive cortical circuits.

'Brainy' children are not cleverer solely by virtue of having more or less grey matter at any one age. Rather, intelligence is related to dynamic properties of cortical maturation.

\section{METHODS}

Subjects. Three hundred and seven unrelated children and adolescents with no personal or family history of psychiatric or neurological disorders were recruited (Supplementary Table 2). All subjects had age-appropriate versions of the Weschler intelligence scales. In 220 subjects, full-scale IQ was estimated from four subtests (vocabulary, similarities, block design and matrix reasoning), and in 87 children two subtests were used (vocabulary and block design). For longitudinal analyses, subjects were divided into three groups on the basis of full-scale IQ with the primary constraint of attaining a roughly equal number of total scans in each group. The groups were: superior intelligence (IQ range 121149), high intelligence (IQ range 109-120) and average intelligence (IQ range $83-108)$. All subjects were scanned at least once; 178 participants (58\%) had at least two scans; $92(30 \%)$ had three or more scans; the mean interscan interval was $\sim 2$ yr.

Neuroimaging. T1-weighted magnetic resonance images $(1.5 \mathrm{~mm}$ axial and $2 \mathrm{~mm}$ coronal slices), acquired using three-dimensional spoiled gradient recalled echo in the steady state on a 1.5-T Signa scanner (General Electric), were registered into standardized space ${ }^{24}$ and corrected for non-uniformity artefacts ${ }^{25}$. The inner and outer cortical surfaces were extracted from tissuesegmented images using deformable models, and non-linearly aligned towards a standard template surface ${ }^{26}$. Cortical thickness was measured in native space millimetres using the linked distance between the pial white and grey matter surfaces at 40,962 vertices throughout the cerebral cortex ${ }^{27}$ (see Supplementary Methods). In order to improve the ability to detect population changes, each cortical thickness map was blurred using a 30-mm surface-based blurring kernel, which respects anatomical boundaries and was chosen to maximize statistical power while minimizing false positives ${ }^{15}$. 
Statistical analysis. Pearson's correlations between IQ and cortical thickness were estimated at each cortical point. Each subject contributed only one scan to maintain independence of data, and efforts were made to ensure a wide age range was covered. Developmental effects were explored by dividing the sample equally into four age groups (called early childhood (age range 3.8-8.4 yr), late childhood (range 8.6-11.7 yr), adolescence (11.8-16.9 yr) and early adulthood (17-29 yr)). Correlations for each of 56 brain subregions were $Z$-transformed, and the difference between the $Z$ scores for each age group, and its significance, was calculated. To correct for the large number of comparisons, a false discovery rate of 0.05 was applied ${ }^{28}$. Gender effects were examined for the entire sample in a similar manner.

To exploit the longitudinal nature of our data set, we used linear mixed-model regression, as this technique permits the inclusion of multiple measurements per person, missing data, and irregular intervals between measurements, thereby increasing statistical power while controlling for within-individual variation ${ }^{29}$. Polynomial models for age effects were compared throughout the cerebral cortex and a cubic model found to provide the best fit, with the exception of anterior temporal cortices where a linear model was appropriate. A cubic model was therefore used to model age effects in the analyses presented. We first examined whether the relationship between IQ and cortical thickness differs with age by regressing cortical thickness at every vertex against IQ, age terms, and the interaction of IQ and age terms. For further exploration of the interaction, we divided the subjects into three IQ groups. This approach loses some power by categorizing a continuous variable, but has the advantage of rendering the results readily interpretable, allowing comparisons between highly intelligent and less intelligent groups. The resulting statistical maps were thresholded to control for multiple comparisons using the false discovery rate (FDR) procedure with $q=0.05$ (refs 28,30 ). An FDR threshold was determined for the statistical model using all $P$ values pooled across all effects included in the model. At every cortical point, $t$-statistics were visualized through projection onto a standard brain template (the map shows the results of the interaction between the cubic age term and IQ groups). Such visualization showed clusters of cortical points that had a significant difference between the intelligence groups in the trajectory of cortical growth. The longitudinal analyses selected and averaged all cortical points within each of these clusters. Graphs illustrating the trajectories were generated using fixed-effects parameter estimates.

To illustrate differences in cortical thickness between the superior and average intelligence groups at different ages, linear mixed-models were run at different centred ages. For example, for age seven years, seven was subtracted from the age at scan acquisition, and this value entered as the age term. $t$-statistics representing the differences in cortical thickness between the two intelligence groups at each age were projected onto brain templates. This analysis represents group differences at each age based on values estimated from developmental curves modelled on all data.

\section{Received 25 October; accepted 29 November 2005.}

1. Spearman, C. 'General intelligence' objectively determined and measured. Am. J. Psychol. 15, 201-293 (1904).

2. Gottfredson, L. S. Why $g$ matters: The complexity of everyday life. Intelligence 24, 79-132 (1997)

3. Deary, I. J., Whalley, L. J., Lemmon, H., Crawford, J. R. \& Starr, J. M. The stability of individual differences in mental ability from childhood to old age: Follow-up of the 1932 Scottish Mental Survey. Intelligence 28, 49-55 (2000).

4. Booth, J. R. et al. Neural development of selective attention and response inhibition. Neuroimage 20, 737-751 (2003)

5. Sowell, E. R. et al. Longitudinal mapping of cortical thickness and brain growth in normal children. J. Neurosci. 24, 8223-8231 (2004).

6. McDaniel, M. Big-brained people are smarter. Intelligence 33, 337-346 (2005)

7. Wilke, M., Sohn, J. H., Byars, A. W. \& Holland, S. K. Bright spots: correlations of gray matter volume with IQ in a normal pediatric population. Neuroimage 20, 202-215 (2003)

8. Frangou, S., Chitins, X. \& Williams, S. C. Mapping IQ and gray matter density in healthy young people. Neuroimage 23, 800-805 (2004)

9. Haier, R. J., Jung, R. E., Yeo, R. A., Head, K. \& Alkire, M. T. Structural brain variation and general intelligence. Neuroimage 23, 425-433 (2004).

10. Kraemer, H. C., Yesavage, J. A., Taylor, J. L. \& Kupfer, D. How can we learn about developmental processes from cross-sectional studies, or can we? Am. J. Psychiatry 157, 163-171 (2000).

11. Giedd, J. N. et al. Brain development during childhood and adolescence: a longitudinal MRI study. Nature Neurosci. 2, 861-863 (1999).

12. Wechsler, D. Manual for the Wechsler Intelligence Scale for Children-Revised (The Psychological Corporation, New York, 1974).

13. O'Donnell, S., Noseworthy, M. D., Levine, B. \& Dennis, M. Cortical thickness of the frontopolar area in typically developing children and adolescents. Neuroimage 24, 948-954 (2005).

14. Kabani, N., Le Goualher, G., MacDonald, D. \& Evans, A. C. Measurement of cortical thickness using an automated 3-D algorithm: a validation study. Neuroimage 13, 375-380 (2001).

15. Lerch, J. P. \& Evans, A. C. Cortical thickness analysis examined through power analysis and a population simulation. Neuroimage 24, 163-173 (2005)

16. Gray, J. R., Chabris, C. F. \& Braver, T. S. Neural mechanisms of general fluid intelligence. Nature Neurosci. 6, 316-322 (2003)

17. Duncan, J. et al. A neural basis for general intelligence. Science $289,457-460$ (2000).

18. Kostovic, I., Judas, M., Rados, M. \& Hrabac, P. Laminar organization of the human fetal cerebrum revealed by histochemical markers and magnetic resonance imaging. Cereb. Cortex 12, 536-544 (2002).

19. Kostovic, I. \& Rakic, P. Developmental history of the transient subplate zone in the visual and somatosensory cortex of the macaque monkey and human brain. J. Comp. Neurol. 297, 441-470 (1990).

20. Yakovlev, P. I. \& Lecours, A. R. in Regional Development of the Brain in Early Life (ed. Minokowski, A.) (Blackwell Scientific, Oxford, 1967).

21. Huttenlocher, P. R. \& Dabholkar, A. S. Regional differences in synaptogenesis in human cerebral cortex. J. Comp. Neurol. 387, 167-178 (1997).

22. Hensch, T. K. Critical period regulation. Annu. Rev. Neurosci. 27, 549-579 (2004).

23. Chugani, H. T., Phelps, M. E. \& Mazziotta, J. C. Positron emission tomography study of human brain functional development. Ann. Neurol. 22, 487-497 (1987)

24. Collins, D. L., Neelin, P., Peters, T. M. \& Evans, A. C. Automatic 3D intersubject registration of MR volumetric data in standardized Talairach space. J. Comput. Assist. Tomogr. 18, 192-205 (1994).

25. Sled, J. G., Zijdenbos, A. P. \& Evans, A. C. A nonparametric method for automatic correction of intensity nonuniformity in MRI data. IEEE Trans. Med. Imaging 17, 87-97 (1998)

26. Zijdenbos, A. P., Forghani, R. \& Evans, A. C. Automatic "pipeline" analysis of 3-D MRI data for clinical trials: application to multiple sclerosis. IEEE Trans. Med. Imaging 21, 1280-1291 (2002)

27. MacDonald, D., Kabani, N., Avis, D. \& Evans, A. C. Automated 3-D extraction of inner and outer surfaces of cerebral cortex from MRI. Neuroimage 12, 340-356 (2000).

28. Benjamini, $Y$. \& Hochberg, Y. Controlling the false discovery rate: a practical and powerful approach to multiple testing. J. R. Stat. Soc. B 57, 289-300 (1995).

29. Pinheiro, J. C. \& Bates, D. M. Mixed-effects Models in S and S-PLUS (Springer, New York, 2000)

30. Genovese, C. R., Lazar, N. A. \& Nichols, T. Thresholding of statistical maps in functional neuroimaging using the false discovery rate. Neuroimage 15 , 870-878 (2002)

Supplementary Information is linked to the online version of the paper at www.nature.com/nature.

Acknowledgements This research was supported by the Intramural Research Program of the National Institutes of Health. We acknowledge the statistical advice of G. Chen and technical assistance from T. Nugent III. The authors thank the children who participated in the study and their families.

Author Contributions P.S. designed and wrote the study with J.R. and J.G., and conducted neuroimaging analyses. J.G. and J.R. directed the project. D.G. conducted longitudinal analyses. L.C. was data manager, and R.L. and N.G. advised on interpretation and analysis. J.L. and A.E. developed cortical thickness analytic tools and J.L. developed software for longitudinal neuroimaging analyses.

Author Information Reprints and permissions information is available at npg.nature.com/reprintsandpermissions. The authors declare no competing financial interests. Correspondence and requests for materials should be addressed to P.S. (shawp@mail.nih.gov). 